\title{
Accuracy of 3-dimensional and 2-dimensional quantitative coronary angiography for predicting physiological significance of coronary stenosis: a FAVOR II substudy
}

\author{
Daixin Ding ${ }^{1,2 \#}$, Junqing Yang ${ }^{3 \#}$, Jelmer Westra ${ }^{4}$, Yundai Chen ${ }^{5}$, Yunxiao Chang ${ }^{1,2}$, Martin Sejr-Hansen ${ }^{4}$, \\ Su Zhang ${ }^{1,2}$, Evald H. Christiansen ${ }^{4}$, Niels R. Holm ${ }^{4}$, Bo Xu ${ }^{6}$, Shengxian Tu ${ }^{1,2}$ \\ ${ }^{1}$ Biomedical Instrument Institute, School of Biomedical Engineering, ${ }^{2}$ Shanghai Med-X Engineering Research Center, Shanghai Jiao Tong \\ University, Shanghai 200030, China; ${ }^{3}$ Department of Cardiology, Guangdong Provincial People's Hospital, Guangzhou 510055, China; ${ }^{4}$ Department \\ of Cardiology, Aarhus University Hospital, Skejby, Denmark; ${ }^{5}$ Department of Cardiology, PLA General Hospital, Beijing 100853, China; ${ }^{6}$ Fu Wai \\ Hospital, National Center for Cardiovascular Diseases, Chinese Academy of Medical Sciences, Beijing 100037, China \\ Contributions: (I) Conception and design: B Xu, S Tu; (II) Administrative support: B Xu, Y Chen-EH Christiansen; (III) Provision of study materials \\ or patients: J Yang, J Westra, Y Chen, EH Christiansen, NR Holm, B Xu; (IV) Collection and assembly of data: D Ding, Y Chang, J Westra; (V) \\ Data analysis and interpretation: D Ding, J Yang, J Westra, Y Chang, M Sejr-Hansen; (VI) Manuscript writing: All authors; (VII) Final approval of \\ manuscript: All authors. \\ \#These authors contributed equally to this work. \\ Correspondence to: Shengxian Tu, PhD, FACC, FESC. Med-X Research Institute, Shanghai Jiao Tong University, No. 1954, Huashan Road, Xuhui \\ District, Shanghai 200030, China. Email: sxtu@sjtu.edu.cn; Bo Xu, MBBS. Fu Wai Hospital, National Center for Cardiovascular Diseases, Chinese \\ Academy of Medical Sciences, A 167, Beilishi Road, Xicheng District, Beijing 100037, China. Email: bxu@citmd.com.
}

Background: Three-dimensional quantitative coronary angiography (3D-QCA) enables reconstruction of a coronary artery in 3D from two angiographic image projections. This study compared the diagnostic accuracy of 3D-QCA vs. 2-dimensional (2D) QCA in predicting physiologically significant coronary stenosis, using fractional flow reserve (FFR) as the reference standard.

Methods: All interrogated vessels in the FAVOR II China study and the FAVOR II Europe-Japan study were assessed by 2D-QCA and 3D-QCA according to standard operating procedures in core laboratories. QCA analysts were blinded to the corresponding FFR values.

Results: A total of 645 vessels from 576 patients with 3D-QCA, 2D-QCA, and FFR were analyzed. Using the conventional cut-off value of $50 \%$ for percent diameter stenosis (DS\%), 3D-QCA was more accurate in predicting FFR $\leq 0.80$ than 2D-QCA [accuracy $74.0 \%$ (95\% CI: 69.9-77.7\%) vs. 64.9\% (95\% CI: 61.368.7\%), difference: $9.1 \%, \mathrm{P}<0.001]$. Sensitivity was higher by 3D-QCA compared with 2D-QCA [69.1\% (95\% CI: 63.0-75.1\%) vs. 47.1\% (95\% CI: 40.5-53.6\%), difference: $22.0 \%, \mathrm{P}<0.001]$ and specificity was similar [76.5\% (95\% CI: 72.5-80.6\%) vs. 74.4\% (95\% CI: 70.2-78.6\%), difference: $2.1 \%, \mathrm{P}=0.40]$. Area under the receiver operating characteristic curve was significantly higher for 3D-QCA than for 2D-QCA [0.81 (95\% CI: 0.77-0.84) vs. 0.66 (95\% CI: 0.62-0.71), $\mathrm{P}<0.001]$.

Conclusions: 3D-QCA demonstrated better diagnostic performance in predicting physiologically significant coronary stenosis compared with 2D-QCA, when FFR was used as the reference standard.

Keywords: Coronary physiology; fractional flow reserve (FFR); ischemia; quantitative coronary angiography (QCA)

Submitted Jun 24, 2019. Accepted for publication Aug 09, 2019.

doi: $10.21037 / \mathrm{cdt} .2019 .09 .07$

View this article at: http://dx.doi.org/10.21037/cdt.2019.09.07 


\section{Introduction}

The degree and extent of a luminal narrowing is frequently used to evaluate the severity of coronary artery stenosis in patients with coronary artery disease (CAD). Coronary artery lesions with $\geq 50 \%$ percent diameter stenosis (DS\%) by quantitative coronary angiography (QCA) are traditionally considered to be significant based on study demonstrating that hyperemic flow starts to decrease with presence of at least $50 \%$ reduction in vessel diameter (1). However, novel diagnostic tools such as fractional flow reserve (FFR) have challenged the use of visual estimation or QCA-based evaluation of coronary stenosis (2-4).

Dedicated 3-dimensional (3D) angiographic reconstruction algorithms were developed to overcome some of the inherited limitations in 2D-QCA analysis such as foreshortening and out-of-plane magnification $(5,6)$. Excellent correlation was reported between 3D-QCA and intravascular imaging including intravascular ultrasound (IVUS) (7) and optical coherence tomography (OCT) (5). Compared with 2D-QCA, 3D-QCA reported better diagnostic performance for predicting physiologically significant coronary stenosis when FFR was used as the reference standard (7-9). Nevertheless, these studies had a relatively small population size and were limited by their retrospective design. The aim of the present study was to compare the diagnostic accuracy of $3 \mathrm{D}$ - and 2D-QCA in a large and prospectively enrolled international population, using FFR as the reference standard.

\section{Methods}

This study reports an ad hoc subanalysis of the FAVOR II China (Functional Diagnostic Accuracy of Quantitative Flow Ratio in Online Assessment of Coronary Stenosis, NCT03191708) and FAVOR II Europe-Japan (Functional Assessment by Various Flow Reconstructions, NCT02959814) studies; two prospective, multicenter studies enrolling a total of 637 patients at 16 centers in the Europe and Asia. Both studies were designed to validate the in-procedure diagnostic accuracy of angiography-based quantitative flow ratio (QFR) $(10,11)$. The study design, main endpoints and inclusion and exclusion criteria were reported elsewhere $(10,11)$. Collection of patient outcome data was not planned in the trial designs.

All FFR traces and angiographic image data were analyzed in core laboratories (CCRF, Beijing, China, CardHemo, Med-X research Institute, Shanghai Jiao Tong
University, Shanghai, China, and the Institute of Clinical Medicine, Aarhus University, Denmark) in a blinded fashion. 3D-QCA, 2D-QCA and FFR data were available for paired analysis in 645 vessels. The study protocol complies with the Declaration of Helsinki and was approved by the institutional review board (FAVOR II China study is 2017-861, for FAVOR II Europe/Japan study is 1-10-72219-16). All patients provided written informed consent.

\section{FFR, 2D-QCA and 3D-QCA analysis}

FFR reading was performed in blinded fashion in the core laboratories using RadiView (St. Jude Medical) as specified in the main study $(10,11)$. The angiographic view with better exposure of the stenosis severity was used for 2D-QCA analysis, using the QAngio XA software package (Medis Medical Imaging Systems, Leiden, The Netherlands). Catheter calibration or isocenter calibration was used.

3D-QCA analysis was performed based on two angiographic image views with $\geq 25^{\circ}$ difference using AngioPlus (Pulse Medical Imaging Technology, Shanghai, China) or QAngio XA 3D (Medis Medical Imaging Systems, Leiden, The Netherlands) that both used the same algorithms for 3D angiographic reconstruction. Automated calibration was used for 3D angiographic reconstruction. For each projection, lumen contours were delineated using the same vessel edge detection algorithm as used in the 2D-QCA analysis. Based on the delineated lumen contours, coronary lumen and reference vessel were reconstructed and anatomical parameters were quantified in $3 \mathrm{D}$. All the QCA measurements were performed by experienced users following standard operation procedures (10-12).

\section{Statistical analysis}

Continuous variables are reported as mean $\pm \mathrm{SD}$, and categorical variables are presented as counts (\%). Normality of the data was tested using the Shapiro-Wilk and Q-Q plots. Bland-Altman plots were used to identify agreement between 2D- and 3D-QCA DS\%. Pearson or Spearman correlation test was used to determine the correlation among variables. Pairwise comparisons between 2Dand $3 \mathrm{D}-\mathrm{QCA}$ DS\% were made by Student $t$-test, and Wilcoxon's rank test was used for comparisons between 2Dand 3D-QCA lesion length, minimum luminal diameter (MLD) and reference diameter. Diagnostic performance estimates by 2D- and 3D-QCA were compared using McNemar's test, generalized score statistics and density 
Table 1 Baseline lesion characteristics by 2-dimensional and 3-dimensional quantitative coronary angiography

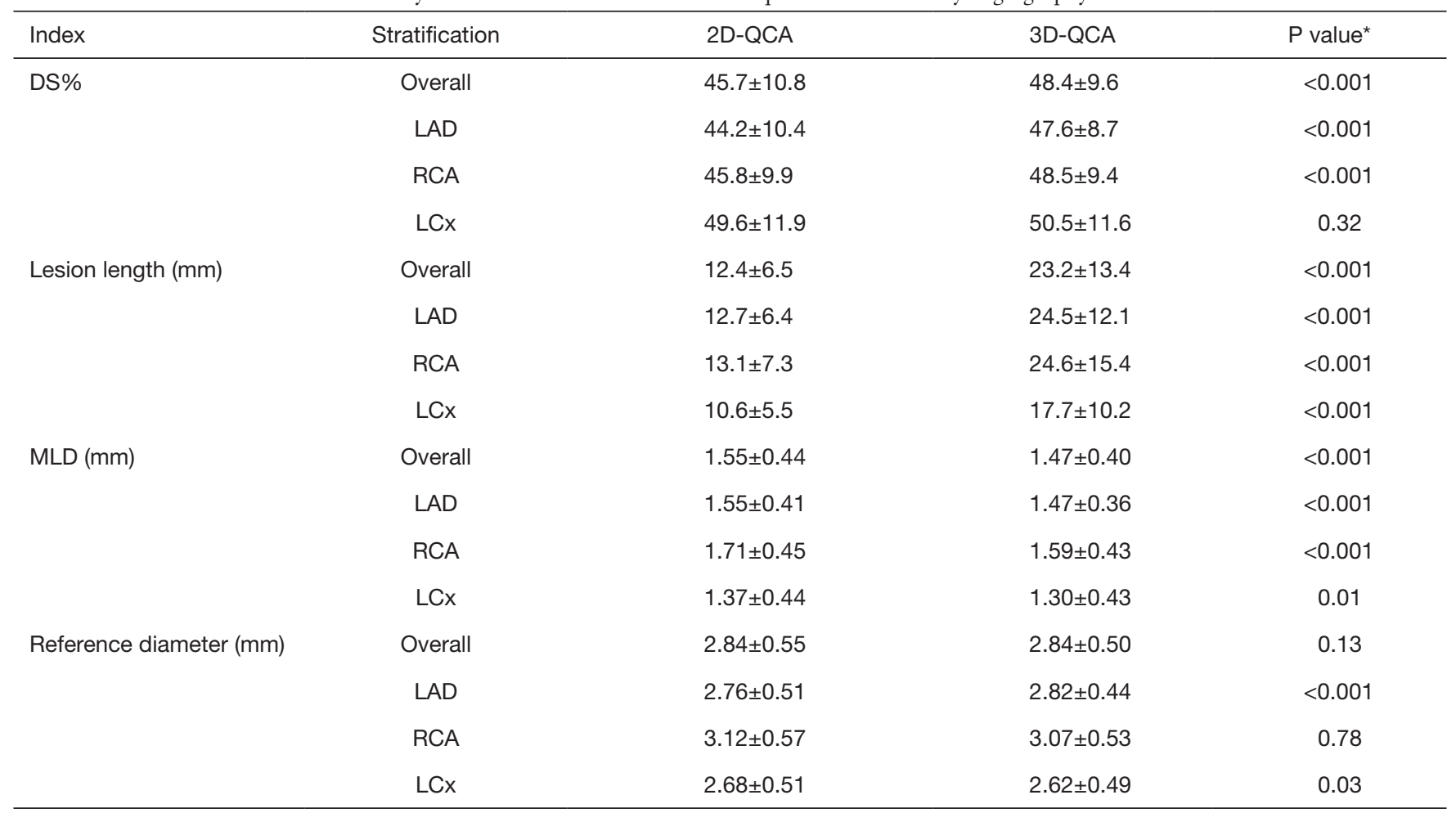

All variables are presented by mean \pm SD. *, DS\% by 3D-QCA and 2D-QCA was compared by paired Student's $t$-test; lesion length, MLD and reference diameter by 3D-QCA and 2D-QCA were compared by Wilcoxon's rank test. 2D-QCA, 2-dimensional quantitative coronary angiography; 3D-QCA, 3-dimensional quantitative coronary angiography; DS\%, percent diameter stenosis; LAD, left anterior descending artery; LCx, left circumflex; MLD, minimum luminal diameter; RCA, right coronary artery.

based logistic regression, as appropriate. Comparisons for accuracy, sensitivity and specificity among groups stratified by vessel were performed using chi-squared test. Area under the receiver-operating characteristic (ROC) curves (AUC) for QCA DS\% to predict FFR $\leq 0.80$ were compared using the Hanley and McNeil method. All statistical analyses were performed using the STATA 15 (StataCorp LLC, Texas, USA) and R software (R Foundation for Statistical Computing, Vienna, Austria). A two-sided P value of $<0.05$ was considered statistically significant.

\section{Results}

A total of 645 vessels from 576 patients with paired 2D-QCA, 3D-QCA and FFR data were available for analysis: $353(55 \%)$ left anterior descending arteries (LAD), 131 (20\%) left circumflex arteries (LCx) and 161 (25\%) right coronary arteries (RCA). Median FFR was 0.85 [interquartile range (IQR): $0.77-0.90$ ] in the overall population. A total of $223(35 \%)$ vessels were hemodynamically significant as defined by FFR $\leq 0.80$. Baseline patient characteristics are listed in Table S1.

\section{Correlation and agreement between $2 D$ - and $3 D-Q C A$ measurement}

Baseline lesion characteristics by 2D- and 3D-QCA were shown in Table 1. Compared with 2D-QCA, 3D-QCA showed higher DS\%, longer lesions, lower MLD but similar reference diameter in the overall population. Correlation was good between 2D-QCA DS\% and 3D-QCA DS\% $(0.59, \mathrm{P}<0.001)$ with modest scatter around the regression line (Figure $1 A$ ). The mean difference was $2.7 \% \pm 9.3 \%$ (Figure 1B).

\section{Correlation between FFR and QCA measurements}

3D-QCA correlated better than 2D-QCA with FFR (-0.56 vs. $-0.32, \mathrm{P}<0.001)$ (Figure 2). Subgroup analysis showed that the difference was statistically significant in LAD $(-0.59$ 

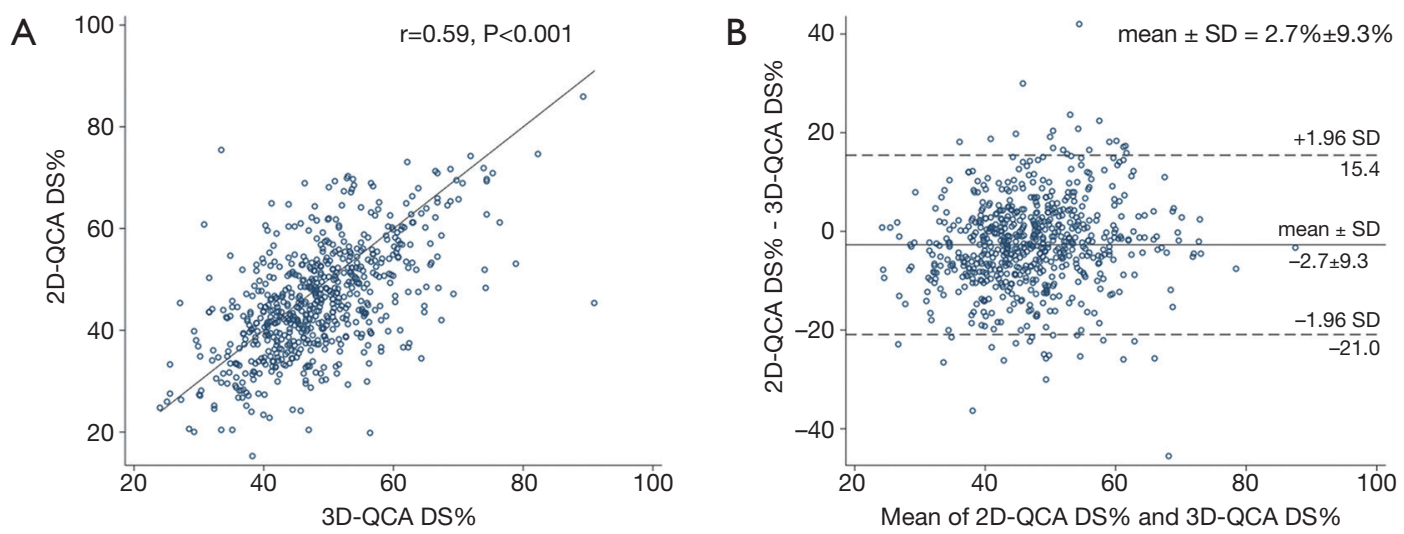

Figure 1 Correlation (A) and agreement (B) between 2D-QCA and 3D-QCA for DS\%. 2D-QCA, 2-dimensional quantitative coronary angiography; 3D-QCA, 3-dimensional quantitative coronary angiography; DS\%, percent diameter stenosis; SD, standard deviation.
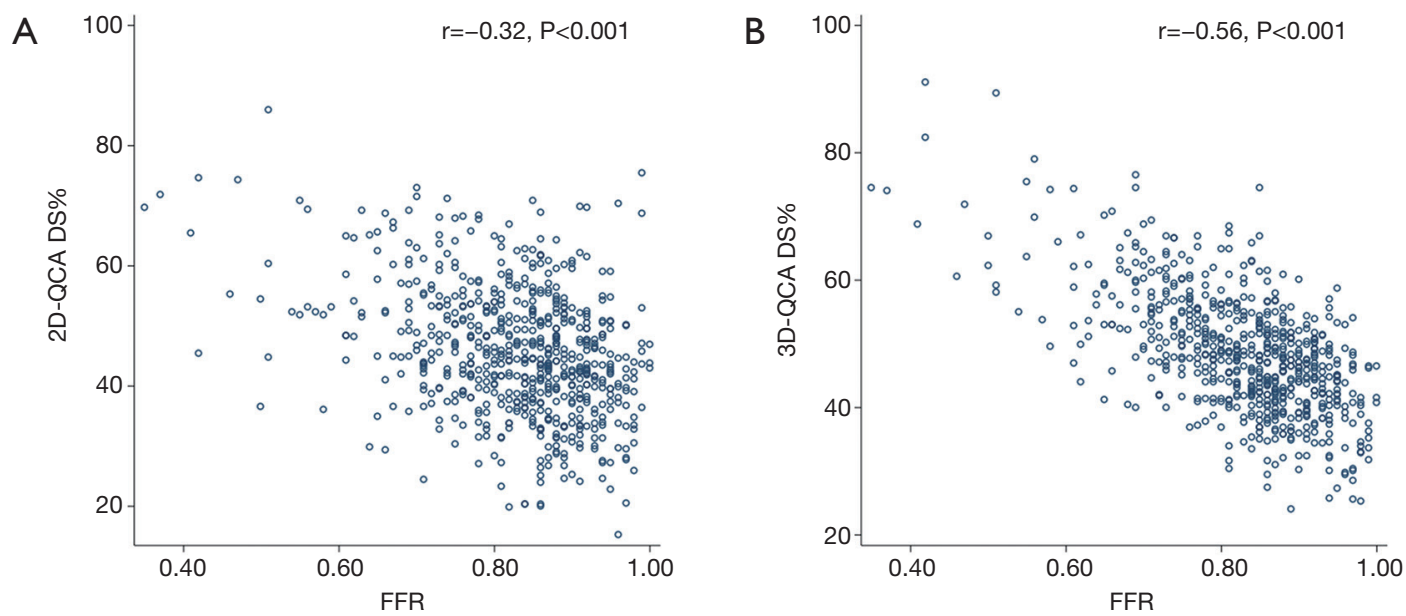

Figure 2 Correlation between DS\% versus FFR for 2D-QCA (A) and 3D-QCA (B). 2D-QCA, 2-dimensional quantitative coronary angiography; 3D-QCA, 3-dimensional quantitative coronary angiography; DS\%, percent diameter stenosis; FFR, fractional flow reserve.

vs. $-0.38, \mathrm{P}<0.001)$, RCA $(-0.66$ vs. $-0.37, \mathrm{P}<0.001)$, and LCx $(-0.66$ vs. $-0.43, \mathrm{P}=0.01)$.

\section{Performance of $2 D$ - and $3 D-Q C A$ in predicting physiologically significant stenosis}

Applying a discrimination limit of DS $\% \geq 50 \%$ to predict FFR $\leq 0.80$ resulted in significantly better diagnostic accuracy $(74.0 \%$ vs. $64.9 \%, \mathrm{P}<0.001)$ and sensitivity (69.1\% vs. $47.1 \%, \mathrm{P}<0.001)$ for $3 \mathrm{D}-\mathrm{QCA}$ compared with 2D-QCA (Tables 2,S2,S3). Using FFR as the reference standard, 3D-QCA DS\% had a higher AUC compared with 2D-QCA DS\% [0.81 (95\% CI: 0.77-0.84) vs. 0.66 (95\%
CI: 0.62-0.71), $\mathrm{P}<0.001]$ (Figure 3).

The relationship between FFR and DS\% category is shown in Figure 4. Lesions were more likely to become physiologically significant with increasing DS\%. Nevertheless, misclassification between physiological significance and anatomical obstruction occurred more with 2D-QCA than 3D-QCA. FFR indicated physiological significance in $21.6 \%$ of lesions with $<40 \%$ DS by $2 \mathrm{D}$-QCA. The false negative ratio decreased to $5.6 \%$ when DS\% was evaluated by $3 \mathrm{D}$-QCA. Likewise, in $27.3 \%$ of lesions with $\geq 70 \%$ DS by 2D-QCA, FFR showed physiological insignificance. This false positive ratio decreased to $6.7 \%$ when DS\% was evaluated by 3D-QCA. 
Table 2 Diagnostic performance of 50\% diameter stenosis by 2-dimensional and 3-dimensional quantitative coronary angiography for predicting fractional flow reserve $\leq 0.80$

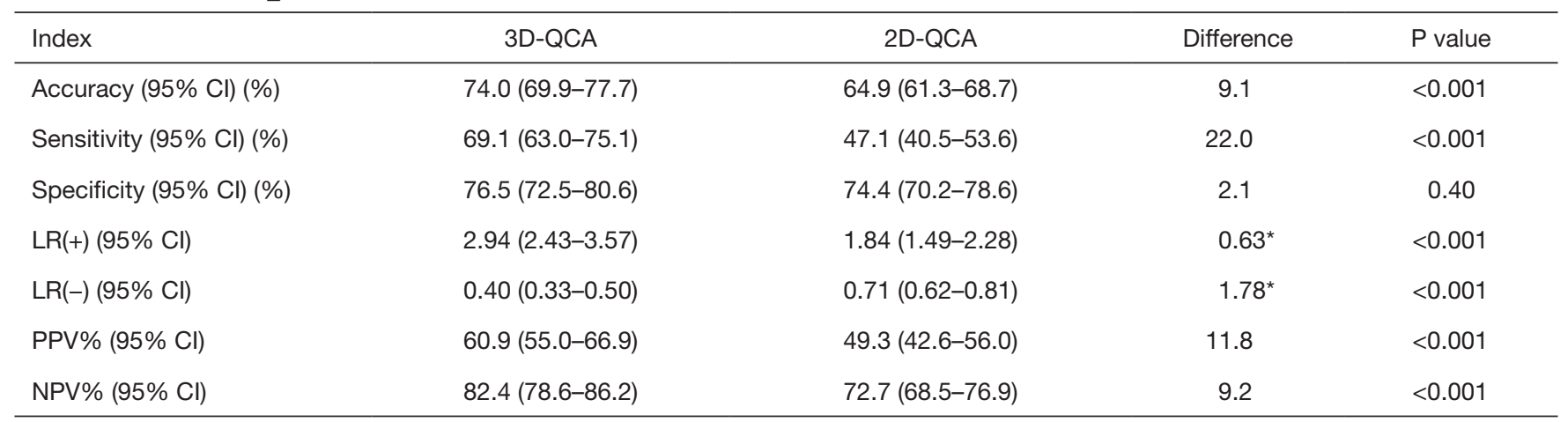

*, ratio. Accuracy, sensitivity and specificity were compared by McNemar's test, PPV and NPV were compared by generalized score statistics, and LR(+) and LR(-) were compared using DLR regression model. 2D-QCA, 2-dimensional quantitative coronary angiography; 3D-QCA, 3-dimensional quantitative coronary angiography; LR(+), positive likelihood ratio; LR(-), negative likelihood ratio; NPV, negative predictive value; PPV, positive predictive value.

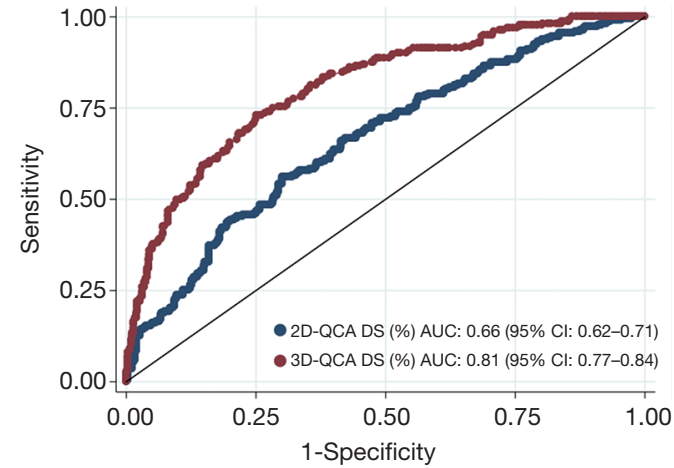

Figure 3 Receiver operating characteristic (ROC) curves of 2D-QCA and 3D-QCA to predict FFR $\leq 0.80$. 2D-QCA, 2-dimensional quantitative coronary angiography; 3D-QCA, 3-dimensional quantitative coronary angiography; DS\%, percent diameter stenosis; AUC, area under the receiver operating characteristic curve.

\section{Influence of lesion location on diagnostic performance}

Median FFR was significantly lower in LAD lesions [0.81 (IQR: 0.75-0.87)] than in RCA lesions [0.88 (IQR: 0.83$0.94), \mathrm{P}<0.001]$ or in LCx lesions [0.88 (IQR: 0.82-0.93), $\mathrm{P}<0.001]$. For both 2D-QCA DS\% and 3D-QCA DS\%, significant variations existed in sensitivity and specificity among lesions in LAD, LCx and RCA (Table 3). The optimal cut-off values for DS\% in predicting FFR $\leq 0.80$ were $50 \%$ for 3D-QCA and $48 \%$ for 2D-QCA (Figure 5 ). Marked variations existed in optimal cut-off values and the associated diagnostic performance estimates after stratification by lesion location (Figure 5). Applying vesselspecific optimal cut-off values in DS\% for both 3D-QCA (cut-off values: $49 \%$ for LAD, $56 \%$ for $\mathrm{LCx}$ and $52 \%$ for RCA) and 2D-QCA (cut-off values: $45 \%$ for LAD, $52 \%$ for LCx and $48 \%$ for RCA), the superiority of 3D-QCA over 2D-QCA remained in diagnostic accuracy $(74.3 \% v s$. $65.1 \%, \mathrm{P}<0.001)$, sensitivity $(73.1 \%$ vs. $56.1 \%, \mathrm{P}<0.001)$ and specificity $(74.9 \%$ vs. $69.9 \%, \mathrm{P}=0.04)$.

\section{Discussion}

This is the largest study to compare the diagnostic accuracy of 2D-QCA and 3D-QCA for the physiological assessment of coronary stenosis in prospectively enrolled patient populations. We observed that (I) 3D-QCA had better diagnostic accuracy than 2D-QCA, with FFR as the reference standard; (II) when evaluated by 2D-QCA, 21.6\% of coronary lesions with DS $<40 \%$ were physiologically significant, and $27.3 \%$ with diameter stenosis $\geq 70 \%$ were physiologically non-significant. The diagnostic discordance with FFR was reduced when stenosis $<40 \%$ and $\geq 70 \%$ DS were evaluated by $3 \mathrm{D}-\mathrm{QCA}$; (III) the optimal cut-off value of DS\% by 3D-QCA to predict physiologically significant coronary stenosis is $50 \%$; (IV) diagnostic performance and optimal diagnostic cut-off value varies in different vessels, and the improvement in diagnostic performance of 3D-QCA over 2D-QCA is greatest in LAD lesions.

The accuracy of $2 \mathrm{D}-\mathrm{QCA}$ in the present study population is moderate due to a number of well-known limitations in 2D analysis, including vessel foreshortening, 

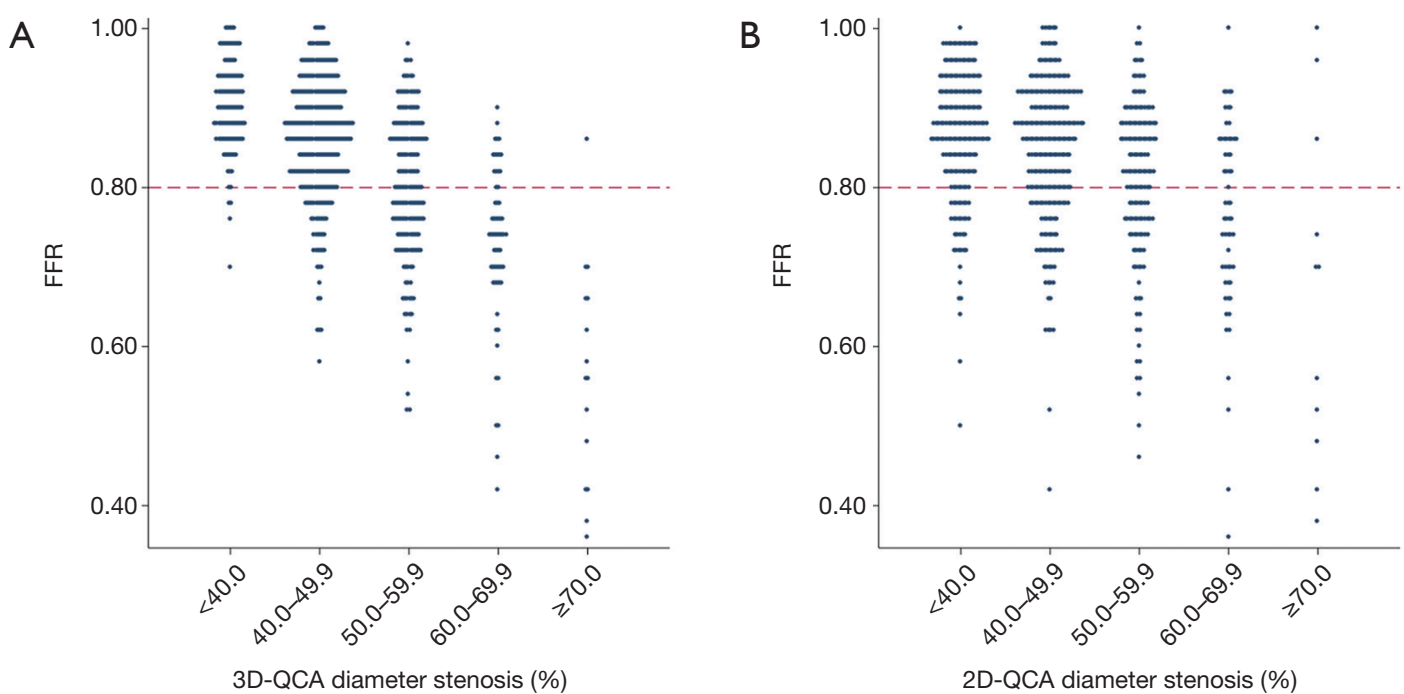

Figure 4 Scatter plot showing relationship between angiographic stenosis grade and FFR for all vessels by 3D-QCA (A) and 2D-QCA (B). The dotted line represents FFR $=0.80$. Numbers in each category: $(A)<40 \%, n=108 ; 40 \%$ to $49.9 \%, n=284 ; 50 \%$ to $59.9 \%$, $n=178 ; 60 \%$ to $69.9 \%, \mathrm{n}=60 ; \geq 70 \%, \mathrm{n}=15$. In lesions graded $<40 \%$ DS, FFR $\leq 0.80$ was present in $5.6 \%$, thus in $5.6 \%$ of stenoses $<40 \%$ DS, FFR indicated physiologically significant lesion. In lesions graded $\geq 70 \%$ DS, FFR $\leq 0.80$ was present in $93.3 \%$, thus in $6.7 \%$ of stenoses $\geq 70 \%$ DS, FFR indicated physiologically non-significant lesion. In lesions graded between $40 \%$ to $69.9 \%$ DS, FFR $\leq 0.80$ was present in $38.9 \%$. (B) $<40 \%$, $\mathrm{n}=194 ; 40 \%$ to $49.9 \%, \mathrm{n}=238 ; 50 \%$ to $59.9 \%, \mathrm{n}=144 ; 60 \%$ to $69.9 \%, \mathrm{n}=58 ; \geq 70 \%, \mathrm{n}=11$. In lesions graded $<40 \%$ DS, FFR $\leq 0.80$ was present in $21.6 \%$, thus in $21.6 \%$ of stenoses $<40 \%$ DS, FFR indicated physiologically significant lesion. In lesions graded $\geq 70 \%$ DS, FFR $\leq 0.80$ was present in $72.7 \%$, thus in $27.3 \%$ of stenoses $\geq 70 \%$ DS, FFR indicated physiologically non-significant lesion. In lesions graded between $40 \%$ to $69.9 \%$ DS, FFR $\leq 0.80$ was present in 39.3\%. 2D-QCA, 2-dimensional quantitative coronary angiography; 3D-QCA, 3-dimensional quantitative coronary angiography; DS\%, percent diameter stenosis; FFR, fractional flow reserve.

Table 3 Stratified analysis of the diagnostic performance of 50\% diameter stenosis by 2-dimensional and 3-dimensional quantitative coronary angiography for predicting fractional flow reserve $\leq 0.80$ according to different lesion locations

\begin{tabular}{|c|c|c|c|c|}
\hline Index & LAD & LCx & $\mathrm{RCA}$ & $P$ value \\
\hline \multicolumn{5}{|c|}{ 2D-QCA 50\% DS } \\
\hline Accuracy & $61.8(56.7-66.9)$ & $66.4(58.2-74.6)$ & 70.8 (63.7-77.9) & 0.13 \\
\hline Sensitivity & $39.6(32.0-47.7)$ & 75.0 (55.1-89.3) & $58.3(40.8-74.5)$ & $<0.001$ \\
\hline Specificity & $79.9(73.6-85.3)$ & $64.1(54.0-73.3)$ & 74.7 (65.8-81.8) & 0.01 \\
\hline \multicolumn{5}{|c|}{ 3D-QCA 50\% DS } \\
\hline Accuracy & 74.5 (70.0-79.1) & $69.4(61.5-77.5)$ & 76.4 (69.8-83.0) & 0.38 \\
\hline Sensitivity & $61.6(53.6-69.2)$ & 85.7 (67.3-96.0) & 88.9 (73.9-96.9) & 0.004 \\
\hline Specificity & $85.1(79.2-89.8)$ & $65.0(55.0-74.2)$ & 72.8 (64.1-80.4) & $<0.001$ \\
\hline
\end{tabular}

Values are $\mathrm{n} \%(95 \% \mathrm{Cl})$. Comparisons for accuracy, sensitivity and specificity among lesion groups of LAD, LCx and RCA were performed by chi-squared test. 2D-QCA, 2-dimensional quantitative coronary angiography; 3D-QCA, 3-dimensional quantitative coronary angiography; DS\%, percent diameter stenosis; LAD, left anterior descending artery; LCx, left circumflex; RCA, right coronary artery. 
A

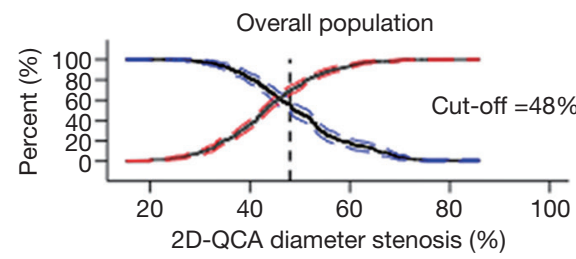

Left circumflex artery

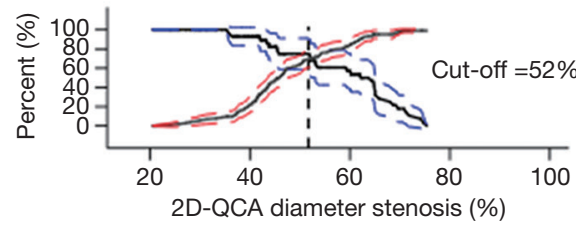

B

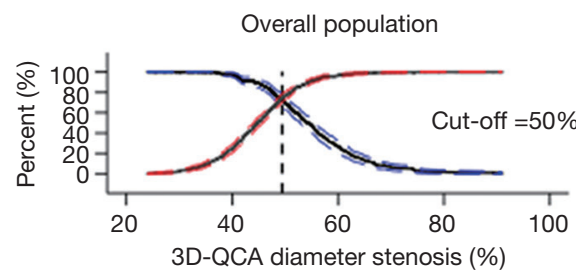

Left circumflex artery

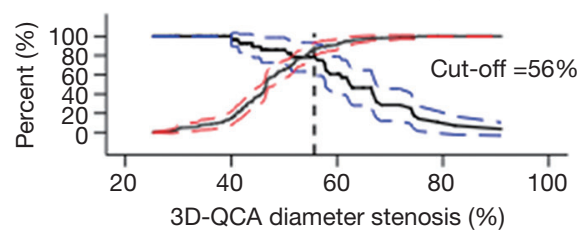

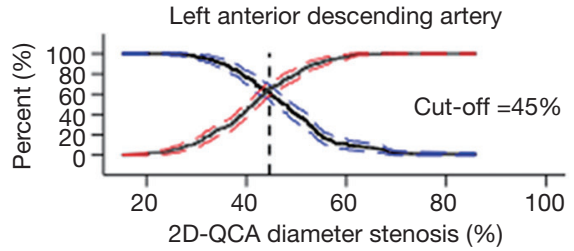
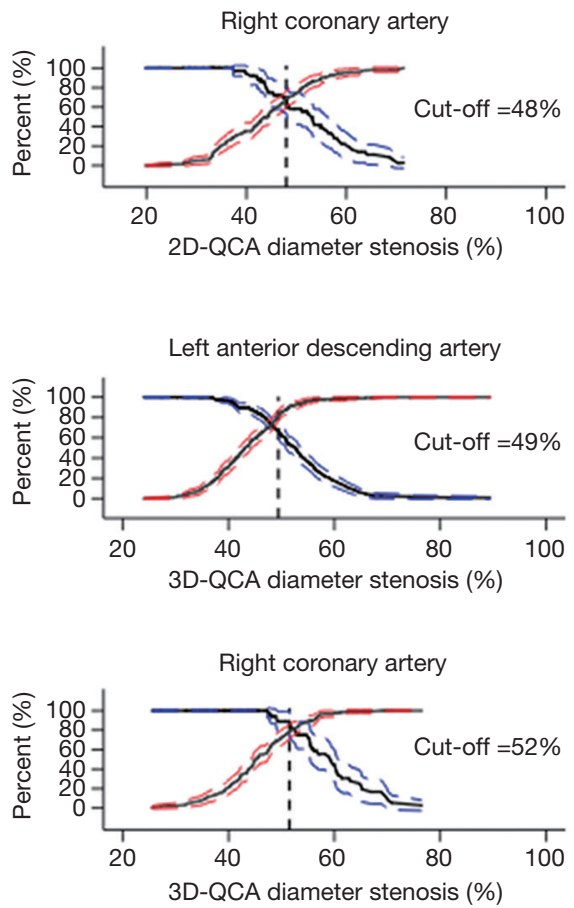

Figure 5 Comparisons of optimal cut-off values of DS\% by 2D-QCA (A) and 3D-QCA (B) among lesions in the overall population, left anterior descending artery, left circumflex and right coronary artery. 2D-QCA, 2-dimensional quantitative coronary angiography; 3D-QCA, 3-dimensional quantitative coronary angiography; DS\%, percent diameter stenosis.

out-of-plane magnification, and imprecise representation of lumen cross-sections for eccentric lesions (13). Our findings are in line with previous studies showing limited diagnostic performance of 2D-QCA indices in predicting physiological significance of coronary stenosis $(2,14)$. By reconstruction of lumen geometry from two angiographic projections, 3D-QCA partially resolved some of these limitations $(13,15,16)$. Of note, Yong et al. previously compared the diagnostic accuracy of 3D-QCA vs. 2D-QCA (8) in predicting FFR and found that MLA by 3D-QCA had higher diagnostic accuracy than by 2D-QCA. However, DS\% by $3 \mathrm{D}-\mathrm{QCA}$ had much lower diagnostic accuracy in predicting FFR $\leq 0.80$ than by $2 \mathrm{D}-\mathrm{QCA}$ (AUC $=0.63 v s$. $0.74)$. On the contrary, we found that DS\% by $3 \mathrm{D}-\mathrm{QCA}$ had substantially higher accuracy in predicting FFR $\leq 0.80$ than by 2D-QCA (AUC $=0.81$ vs. 0.66). The difference might be explained by the difference in the study design and the enrolled patient populations. While the study by Yong et al. was a retrospective study with a population of only 63 patients, the present study analyzed 576 unselected patients with indication for coronary physiological assessment who were prospectively enrolled in 16 international centers. The prospective study design and large patient population may also partly contribute to the result that the diagnostic accuracy of 3D-QCA in our study tends to be higher than in other studies which investigate its diagnostic performance (9). Using this large patient population, we also showed that the superiority in diagnostic accuracy by 
3D-QCA was maintained in the three major epicardial coronary arteries. In addition, we demonstrated for the first time that 3D-QCA on average yielded a smaller MLD but similar reference vessel size compared with 2D-QCA. This resulted in a higher diameter stenosis by $3 \mathrm{D}-\mathrm{QCA}$ than by 2D-QCA. Indeed, 2D-QCA allows assessment of lesion severity in a single projection that might not fully expose the lesion severity. On the contrary, 3D-QCA reconstructed the coronary lumen in $3 \mathrm{D}$ and hence increased the chance of exposing the smaller lumen diameter. This improved assessment of the narrowest cross-sections may have contributed to improved accuracy in predicting physiologically significant coronary stenosis.

Although previous studies demonstrated discrepancies between FFR and angiographic diameter stenosis $(2,4,7,8)$, in daily practice, decisions about revascularization are mainly based on angiographic DS\% in combination with other factors related to ischemia (17). Solely considering angiographic DS\%, it is generally perceived by many interventional cardiologists that lesions of $<40 \%$ DS are unlikely to reduce hyperemic blood flow, while lesions of $>70 \%$ DS are believed to cause ischemia. The recent RIPCORD (4) study found that $13 \%$ of the lesions with $<30 \%$ DS by visual assessment had an FFR $\leq 0.80$, while $47 \%$ of the lesions with $>70 \%$ DS had an FFR $>0.80$ (4). The present study was based on data from prospectively enrolled consecutive patients with coronary lesions between $30 \%$ and $90 \%$ DS. The diagnostic performance was improved by $3 \mathrm{D}-\mathrm{QCA}$ compared to $2 \mathrm{D}$-QCA. The increased rates of false negative predictions by 2D-QCA could be explained by several reasons mainly related to diffused atherosclerosis and proximal lesion subtending large myocardial territories in which blood flow can be limited even without critical stenosis by angiography (18). The lower false negative ratio in the RIPCORD study compared with that of 2D-QCA in our study is in line with some previous studies showing that visual assessment of angiography had a better diagnostic performance than $2 \mathrm{D}$-QCA in predicting FFR $\leq 0.80$ because physicians take additional clinical factors related to ischemia into account (17). The false positive ratio was much lower by 2D-QCA in this study than in the RIPCORD study, with that of $3 \mathrm{D}-\mathrm{QCA}$ being even lower. This gap may be explained by the tendency towards overestimation of stenosis severity by physician's visual assessment than by QCA $(14,17,19,20)$. Of note, the false negative ratio and false positive ratio were much smaller for 3D-QCA than for 2D-QCA, again suggesting the superiority of 3D-QCA in identifying physiological significance of coronary stenosis.

Revascularization of physiologically non-significant stenosis introduces procedural risk without offering comparable benefits (3), while deferral of physiologically significant stenosis can result in detrimental clinical consequences (21). The discrepancies between FFR and QCA reveal the theoretical differences between these two techniques. While angiography depicts the degree and extent of epicardial luminal narrowing, FFR aims to reveal to what extent coronary flow is limited by an epicardial stenosis (22). Reliability of FFR may be limited as maximum hyperemia may be difficult to achieve due to complex patientspecific physiological conditions such as high venous pressure and the presence of microvascular dysfunction (23). There are situations in which different coronary flow patterns with identical anatomical components are not differentiated by QCA (24). Technically, both FFR and QCA have inherent limitations. The quality of FFR measurement may be hampered by several pitfalls (e.g., dampening, drift, waveform distortion) (25) while QCA is vulnerable to bias related to lumen border detection, especially for severe lesions. Therefore, one-to-one correspondence between FFR and QCA values is not expected. And this precludes the use of 2D-QCA or 3D-QCA alone to guide revascularization decision-making. Nevertheless, it is promising that 3D-QCA appears to identify physiologically significant coronary stenosis better than 2D-QCA.

In this study, the overall optimal cut-off value for 3D-QCA DS\% was equal to the traditional cut-off value of $50 \%$ that thus appears appropriate. This is in accordance with a recent study by Toth $e t a l$. in which the overall diagnostic performance of QCA DS\% decreased when adjusting the cut-off value from $50 \%$ to $70 \%$ (2). However, our subgroup analysis found that the optimal cut-off value was lowest for LAD lesions while highest for LCx lesions by both 2D- and 3D-QCA. Previous studies have found that FFR tends to be lower in segments subtending larger myocardial mass than in segments subtending smaller myocardium territories $(2,22,26)$. This is further confirmed by our study where median FFR in LAD lesions was lower compared to non-LAD lesions, and may explain the low sensitivity and high specificity for $50 \%$ DS in LAD vs. non-LAD lesions. We also found that for both 2D- and 3D-QCA, no statistically significant differences 
exist in the diagnostic accuracy in predicting FFR $\leq 0.80$ among lesions in LAD, LCx and RCA, despite significant variations in sensitivity and specificity. This indicates that although there may be dissimilarities in the physiological environment combined with variations in vessel length and bifurcation conditions in individual vessels (27), the overall discriminative power for physiologically significant lesions of QCA remains stable in major branch subgroups. Thus, vessel-specific cut-off values for QCA-based endpoints in clinical studies may exert limited benefits. This is further confirmed by our study findings: using vessel-specific cut-off values instead of the same cut-off value in DS\% led to higher sensitivity $(73.1 \%$ vs. $69.1 \%)$ but lower specificity $(74.9 \%$ vs. $76.5 \%$ ) for $3 \mathrm{D}-\mathrm{QCA}$ in predicting physiologically significant stenosis. However, the overall diagnostic accuracy was similar $(74.3 \%$ vs. $74.0 \%)$.

The anatomy derived from 3D-QCA may be combined with information of blood flow for predicting physiologically significant stenosis. QFR, a novel approach for fast computation of virtual FFR based on 3D-QCA and thrombolysis in myocardial infarction (TIMI) frame count, has been validated against FFR with good diagnostic accuracy in several studies $(10,11,28,29)$. Similar concepts were also tested in other angiography-based FFR methods $(30,31)$.

This study is not without limitation. Selection variation may exist in the 2D-QCA procedure. For every patient, the angiographic image for 2D-QCA analysis was chosen by operator's preference from two angiographic image runs used for 3D-QCA analysis. Nevertheless, the chosen angiographic image runs were with minimum overlap or foreshortening, best image quality and exposure of target vessels, thus reducing the selection variation for $2 \mathrm{D}-\mathrm{QCA}$ to minimum.

\section{Conclusions}

3D-QCA analysis demonstrated higher diagnostic accuracy in predicting physiologically significant coronary stenosis than 2D-QCA analysis. When FFR is not available, 3D-QCA may be used to assist evaluation of obstructive coronary stenosis.

\section{Acknowledgments}

Funding: This work was supported by the Natural Science
Foundation of China (Grant Number 81871460); Shanghai Jiao Tong University (Grant Number YG2016ZD09); and by the Program of Shanghai Technology Research Leader.

\section{Footnote}

Conflicts of Interest: S Tu received a research grant from Medis medical imaging and Pulse medical imaging technology. N Holm received research grants from Medis medical imaging, Abbot, and Boston Scientific. The other authors have no conflicts of interest to declare.

Ethical Statement: The authors are accountable for all aspects of the work in ensuring that questions related to the accuracy or integrity of any part of the work are appropriately investigated and resolved. The study protocol complies with the Declaration of Helsinki and was approved by the institutional review board (FAVOR II China study is 2017-861, for FAVOR II Europe/Japan study is 1-10-72219-16). All patients provided written informed consent.

\section{References}

1. Gould KL, Lipscomb K, Hamilton GW. Physiologic basis for assessing critical coronary stenosis. Instantaneous flow response and regional distribution during coronary hyperemia as measures of coronary flow reserve. Am J Cardiol 1974;33:87-94.

2. Toth G, Hamilos M, Pyxaras S, et al. Evolving concepts of angiogram: fractional flow reserve discordances in 4000 coronary stenoses. Eur Heart J 2014;35:2831-8.

3. Tonino PA, De Bruyne B, Pijls NH, et al. Fractional flow reserve versus angiography for guiding percutaneous coronary intervention. N Engl J Med 2009;360:213-24.

4. Curzen N, Rana O, Nicholas Z, et al. Does routine pressure wire assessment influence management strategy at coronary angiography for diagnosis of chest pain? The RIPCORD study. Circ Cardiovasc Interv 2014;7:248-55.

5. Tu S, Xu L, Ligthart J, et al. In vivo comparison of arterial lumen dimensions assessed by co-registered threedimensional (3D) quantitative coronary angiography, intravascular ultrasound and optical coherence tomography. Int J Cardiovasc Imaging 2012;28:1315-27.

6. Tu S, Echavarria-Pinto M, von Birgelen C, et al. Fractional flow reserve and coronary bifurcation anatomy: a novel quantitative model to assess and report the stenosis 
severity of bifurcation lesions. JACC Cardiovasc Interv 2015;8:564-74.

7. Nishi T, Kitahara H, Fujimoto Y, et al. Comparison of 3-dimensional and 2-dimensional quantitative coronary angiography and intravascular ultrasound for functional assessment of coronary lesions. J Cardiol 2017;69:280-6.

8. Yong AS, Ng AC, Brieger D, et al. Three-dimensional and two-dimensional quantitative coronary angiography, and their prediction of reduced fractional flow reserve. Eur Heart J 2011;32:345-53.

9. Chu M, Dai N, Yang J, et al. A systematic review of imaging anatomy in predicting functional significance of coronary stenoses determined by fractional flow reserve. Int J Cardiovasc Imaging 2017;33:1-16.

10. Westra J, Andersen BK, Campo G, et al. Diagnostic performance of in-procedure angiography-derived quantitative flow reserve compared to pressure-derived fractional flow reserve: the FAVOR II Europe/Japan Study. J Am Heart Assoc. doi: 10.1161/JAHA.118.009603.

11. Xu B, Tu S, Qiao S, et al. Diagnostic accuracy of angiography-based quantitative flow ratio measurements for online assessment of coronary stenosis. J Am Coll Cardiol 2017;70:3077-87.

12. Tuinenburg JC, Koning G, Hekking E, et al. One core laboratory at two international sites, is that feasible? An inter-core laboratory and intra-observer variability study. Catheter Cardiovasc Interv 2002;56:333-40.

13. Tu S, Koning G, Jukema W, et al. Assessment of obstruction length and optimal viewing angle from biplane X-ray angiograms. Int J Cardiovasc Imaging 2010;26:5-17.

14. Park SJ, Kang SJ, Ahn JM, et al. Visual-functional mismatch between coronary angiography and fractional flow reserve. JACC Cardiovasc Interv 2012;5:1029-36.

15. Ramcharitar S, Daeman J, Patterson M, et al. First direct in vivo comparison of two commercially available threedimensional quantitative coronary angiography systems. Catheter Cardiovasc Interv 2008;71:44-50.

16. Collet C, Onuma Y, Cavalcante R, et al. Quantitative angiography methods for bifurcation lesions: a consensus statement update from the European Bifurcation Group. EuroIntervention 2017;13:115-23.

17. Adjedj J, Xaplanteris P, Toth G, et al. Visual and quantitative assessment of coronary stenoses at angiography versus fractional flow reserve: the impact of risk factors. Circ Cardiovasc Imaging 2017;10:e006243.

18. De Bruyne B, Hersbach F, Pijls NH, et al. Abnormal epicardial coronary resistance in patients with diffuse atherosclerosis but "Normal" coronary angiography.
Circulation 2001;104:2401-6.

19. Nallamothu BK, Spertus JA, Lansky AJ, et al. Comparison of clinical interpretation with visual assessment and quantitative coronary angiography in patients undergoing percutaneous coronary intervention in contemporary practice: the Assessing Angiography (A2) project. Circulation 2013;127:1793-800.

20. Zhang $\mathrm{H}, \mathrm{Mu} \mathrm{L}, \mathrm{Hu} \mathrm{S}$, et al. Comparison of physician visual assessment with quantitative coronary angiography in assessment of stenosis severity in China. JAMA Intern Med 2018;178:239-47.

21. De Bruyne B, Pijls NH, Kalesan B, et al. Fractional flow reserve-guided PCI versus medical therapy in stable coronary disease. N Engl J Med 2012;367:991-1001.

22. De Bruyne B, Sarma J. Fractional flow reserve: a review. Heart 2008;94:949-59.

23. Meuwissen M, Chamuleau SA, Siebes M, et al. Role of variability in microvascular resistance on fractional flow reserve and coronary blood flow velocity reserve in intermediate coronary lesions. Circulation 2001;103:184-7.

24. Gould KL, Johnson NP, Bateman TM, et al. Anatomic versus physiologic assessment of coronary artery disease: role of coronary flow reserve, fractional flow reserve, and positron emission tomography imaging in revascularization decision-making. J Am Coll Cardiol 2013;62:1639-53.

25. Matsumura M, Johnson NP, Fearon WF, et al. Accuracy of fractional flow reserve measurements in clinical practice: observations from a core laboratory analysis. JACC Cardiovasc Interv 2017;10:1392-401.

26. Leone AM, De Caterina AR, Basile E, et al. Influence of the amount of myocardium subtended by a stenosis on fractional flow reserve. Circ Cardiovasc Interv 2013;6:29-36.

27. Svanerud J, Ahn JM, Jeremias A, et al. Validation of a novel non-hyperemic index of coronary artery stenosis severity: the Resting Full-cycle Ratio (VALIDATE RFR) study. EuroIntervention 2018;14:806-14.

28. Tu S, Westra J, Yang J, et al. Diagnostic accuracy of fast computational approaches to derive fractional flow reserve from diagnostic coronary angiography: the international multicenter FAVOR Pilot Study. JACC Cardiovasc Interv 2016;9:2024-35.

29. Westra J, Tu S, Winther S, et al. Evaluation of coronary artery stenosis by quantitative flow ratio during invasive coronary angiography: The WIFI II Study (WireFree Functional Imaging II). Circ Cardiovasc Imaging 2018;11:e007107. 
30. Pellicano M, Lavi I, De Bruyne B, et al. Validation study of image based fractional flow reserve during coronary angiography. Circ Cardiovasc Interv 2017. doi: 10.1161/ CIRCINTERVENTIONS.116.005259.

31. Morris PD, Ryan D, Morton AC, et al. Virtual

Cite this article as: Ding D, Yang J, Westra J, Chen Y, Chang Y, Sejr-Hansen M, Zhang S, Christiansen EH, Holm NR, Xu B, Tu S. Accuracy of 3-dimensional and 2-dimensional quantitative coronary angiography for predicting physiological significance of coronary stenosis: a FAVOR II substudy. Cardiovasc Diagn Ther 2019;9(5):481-491. doi: 10.21037/cdt.2019.09.07 fractional flow reserve from coronary angiography: modeling the significance of coronary lesions: results from the VIRTU-1 (VIRTUal Fractional Flow Reserve From Coronary Angiography) study. JACC Cardiovasc Interv 2013;6:149-57. 


\section{Supplementary}

Table S1 Baseline characteristics of the study population.

\begin{tabular}{lc}
\hline Characteristics & Value $(\mathrm{n}=576)$ \\
\hline Age, years & $63.8 \pm 10.7$ \\
Male & $73.1[421]$ \\
BMI, kg/m² & $26.1 \pm 4.1\left(\mathrm{n}=569^{\star}\right)$ \\
Current smoker & $24.5[141]$ \\
Hypertension & $66.7[384]$ \\
Hyperlipidemia & $56.4[325]$ \\
Diabetes mellitus & $28.3[163]$ \\
Family history of CAD & $21.5[124]$ \\
Previous PCl & $30.0[173]$ \\
Previous CABG & $2.1[12]$ \\
Clinical presentation & \\
Silent ischemia & $3.3[19]$ \\
Stable angina pectoris & $15.1[87]$ \\
Unstable angina pectoris & $45.1[260]$ \\
Acute myocardial infarction & $35.1[202]$ \\
Other & $1.4[8]$ \\
\hline
\end{tabular}

Values are presented as mean \pm SD or $\%[n] .{ }^{*}$, number of patients for whom continuous variables were calculated. BMI, body mass index; CABG, coronary artery bypass surgery; $\mathrm{CAD}$, coronary artery disease; $\mathrm{PCI}$, percutaneous coronary interventions.
Table S2 Diagnostic accuracy of 2D-QCA in predicting FFR $\leq 0.80$

\begin{tabular}{lccc}
\hline \multirow{2}{*}{$2 \mathrm{D}-\mathrm{QCA} D S \%$} & \multicolumn{2}{c}{ FFR } & Total \\
\cline { 2 - 3 } & $\leq 0.80$ & $>0.80$ & \\
\hline $50 \%$ & 105 & 108 & 213 \\
$<50 \%$ & 118 & 314 & 432 \\
Total & 223 & 422 & 645 \\
\hline
\end{tabular}

2*2 table for DS\% vs. FFR for 2D-QCA. 2D-QCA, 2-dimensional quantitative coronary angiography; DS\%, percent diameter stenosis; FFR, fractional flow reserve.

Table S3 Diagnostic accuracy of 3D-QCA in predicting FFR $\leq 0.80$

\begin{tabular}{lrrl}
\hline \multirow{2}{*}{ 3D-QCA DS\% } & \multicolumn{2}{c}{ FFR } & Total \\
\cline { 2 - 3 } & $\leq 0.80$ & $>0.80$ & \\
\hline$\geq 50 \%$ & 154 & 99 & 253 \\
$<50 \%$ & 69 & 323 & 392 \\
Total & 223 & 422 & 645 \\
\hline
\end{tabular}

$2{ }^{\star} 2$ table for DS\% vs. FFR for 3D-QCA. 3D-QCA, 3-dimensional quantitative coronary angiography; DS\%, percent diameter stenosis; FFR, fractional flow reserve. 